6. Simon F, Mura M, Pagès F, Morand G, Truc P, Louis F, et al. Urban transmission of human African trypanosomiasis, Gabon. Emerg Infect Dis. 2012;18:165-7. http://dx.doi.org/10.3201/eid1801.111384

7. Migchelsen SJ, Büscher P, Hoepelman AI, Schallig HD, Adams ER Human African trypanosomiasis: a review of non-endemic cases in the past 20 years. Int J Infect Dis. 2011;15:e517-24. http://dx.doi.org/10.1016/j.ijid.2011.03.018

8. Checchi F, Funk S, Chandramohan D, Haydon DT, Chappuis F. Updated estimate of the duration of the meningo-encephalitic stage in Gambiense human African trypanosomiasis. BMC Res Notes. 2015;8:292. http://dx.doi.org/10.1186/s13104-015-1244-3

9. Lefterova MI, Suarez CJ, Banaei N, Pinsky BA. Next-generation sequencing for infectious disease diagnosis and management: a report of the Association for Molecular Pathology. J Mol Diagn. 2015;17:623-34. http://dx.doi.org/10.1016/j.jmoldx.2015.07.004

Address for correspondence: Wenhong Zhang, Huashan Hospital, Department of Infectious Diseases, Fudan University, 12 M. Wulumuqi Rd, Shanghai 200040, China; email: zhangwenhong@fudan.edu.cn

\title{
Dengue-Associated Posterior Reversible Encephalopathy Syndrome, Vietnam
}

\section{Nguyen Thi Hoang Mai, Nguyen Hoan Phu, Ho Dang Trung Nghia, Tran My Phuong, Du Trong Duc, Nguyen Van Vinh Chau, Bridget Wills, Choie Cheio Tchoyoson Lim, Guy Thwaites, Cameron Paul Simmons, Sophie Yacoub}

\begin{abstract}
Author affiliations: Hospital for Tropical Diseases, Ho Chi Minh City, Vietnam (N.T.H. Mai, N.H. Phu, H.D.T. Nghia, T.M. Phuong, D.T. Duc, N.V.V. Chau); Oxford University Clinical Research Unit, Wellcome Trust Major Overseas Programme, Ho Chi Minh City (N.T.H. Mai, N.V.V. Chau, B. Wills, G. Thwaites, C.P. Simmons, S. Yacoub); University of Oxford, Oxford, UK (B. Wills, G. Thwaites); National Neuroscience Institute, Singapore (C.C.T. Lim); University of Melbourne, Melbourne, Victoria, Australia (C.P. Simmons); Imperial College London, Hammersmith Campus, London, UK (S. Yacoub)
\end{abstract}

DOI: https://doi.org/10.3201/eid2402.171634

Dengue can cause neurologic complications in addition to the more common manifestations of plasma leakage and coagulopathy. Posterior reversible encephalopathy syndrome has rarely been described in dengue, although the pathophysiology of endothelial dysfunction likely underlies both. We describe a case of dengue-associated posterior reversible encephalopathy syndrome and discuss diagnosis and management.
$\mathrm{O}^{\prime \prime}$ December 20, 2015, a 55-year-old woman in Vietnam sought medical care at her local hospital near Ho Chi Minh City with 1 day of fever, muscle aches, and anorexia. She had no remarkable medical history; she did not take any medications and had not received any recent vaccinations. Initial hematology and biochemistry tests were normal, but a rapid test for dengue nonstructural protein 1 antigen was positive. She remained hemodynamically stable and did not experience any bleeding or have evidence of plasma leakage. On December 24, she had a generalized convulsion and was transferred to the intensive care unit at the Hospital for Tropical Diseases.

At admission, her Glasgow Coma Scale score was $10 / 15$ (eyes 3 , motor 4 , voice 3 ); she was confused, and speech was slurred. Her temperature was $37.5^{\circ} \mathrm{C}$, heart rate was 100 beats $/ \mathrm{min}$, blood pressure was $140 / 90$ $\mathrm{mmHg}$, and respiratory rate was 18 breaths $/ \mathrm{min}$. Cardiorespiratory and abdominal examinations were normal. Neurologic examination demonstrated increased tone in upper and lower limbs and bilateral upgoing plantar reflexes; assessment of power was difficult because of generalized rigidity. Examination of her eye movements demonstrated vertical gaze palsy, but other cranial nerves were intact.

Blood tests showed hemoglobin level of $14 \mathrm{~g} / \mathrm{dL}$, hematocrit level of $42.2 \%$, a white cell count of $11 \times 10^{9}$ cells $/ \mathrm{L}$, and a platelet count of $100 \times 10^{9} / \mathrm{L}$. A test for dengue nonstructural protein 1 antigen remained positive, but PCR was negative. Urea and electrolyte levels were normal, but liver transaminases showed elevated aspartate aminotransferase of $386 \mathrm{U} / \mathrm{L}$ and alanine aminotransferase of $242 \mathrm{U} / \mathrm{L}$. Chest radiograph was unremarkable, but a brain computed tomography scan demonstrated bilateral cerebral and cerebellar white matter hypodensities. Lumbar puncture demonstrated an opening pressure of 12 $\mathrm{cm} \mathrm{H}_{2} \mathrm{O}$, a cerebrospinal fluid (CSF) cell count of 7 cells/ $\mu \mathrm{L}$, a red cell count of 4 cells $/ \mu \mathrm{L}$, protein level of $4.4 \mathrm{~g} /$ $\mathrm{dL}$, glucose level of $4.76 \mathrm{mmol} / \mathrm{L}(10.4 \mathrm{mmol} / \mathrm{L}$ in blood), and lactate level of $3.25 \mathrm{mmol} / \mathrm{L}$. Dengue virus IgM was detected in CSF; Japanese encephalitis virus IgM was negative. CSF dengue and herpes simplex virus PCR were negative. Microbiologic cultures performed on blood and CSF were sterile.

Magnetic resonance imaging (MRI) of the brain, performed the following day, demonstrated bilateral symmetric high signal on T2-weighted and fluid attenuation inversion recovery images, involving periventricular and deep cerebral white matter (Figure, panel A). The differential diagnoses included encephalitis or acute demyelinating encephalomyelitis (ADEM), and a course of intravenous methylprednisolone was started $(1 \mathrm{~g} / \mathrm{d})$. After 5 days, this regimen was converted to oral prednisolone $(60 \mathrm{mg} / \mathrm{d})$, tapering over 5 days. Phenobarbital was administered for 1 

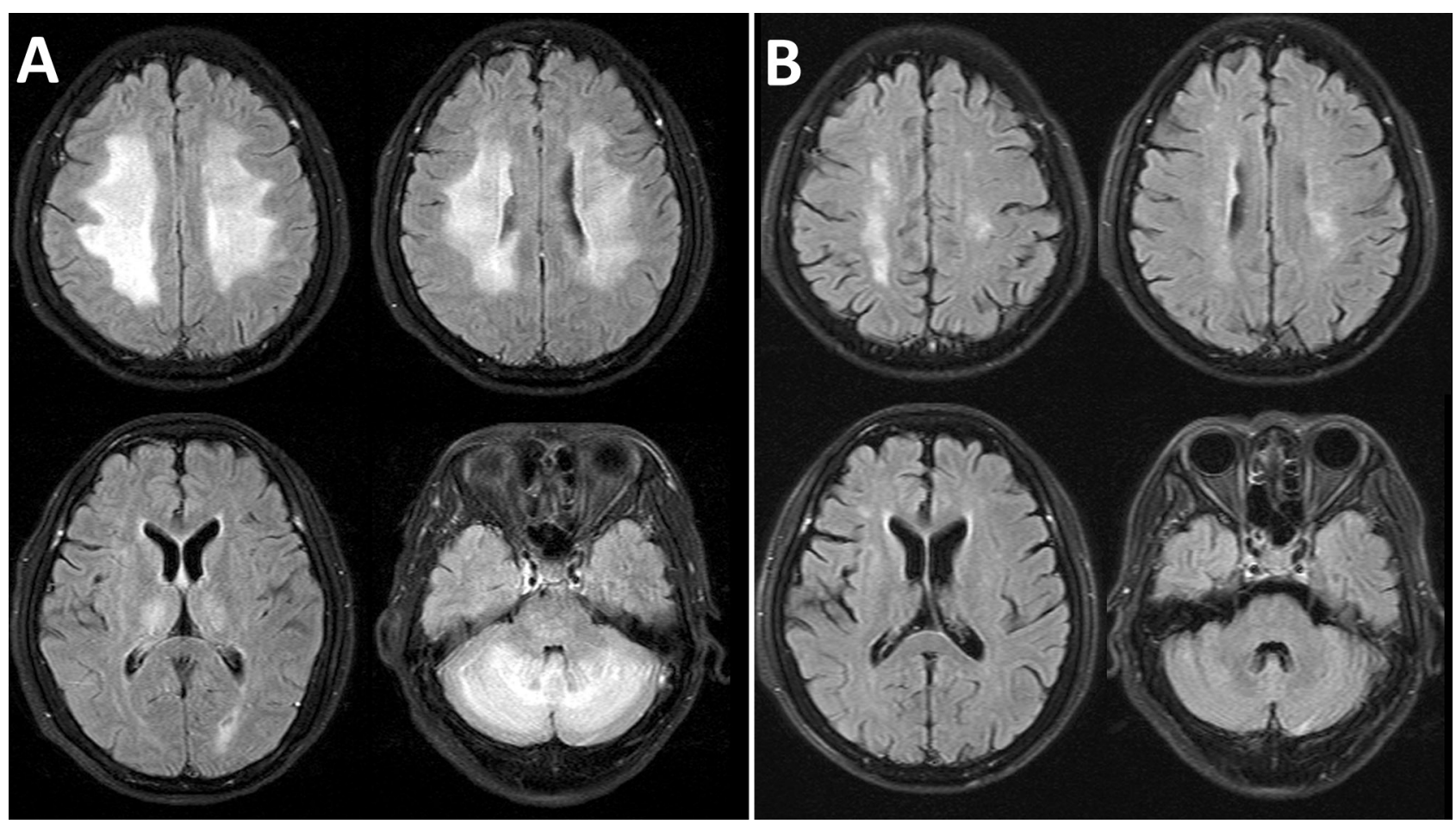

Figure. Fluid-attenuated inversion recovery magnetic resonance images of the brain of a 55-year-old woman with dengue-associated posterior reversible encephalopathy syndrome, Ho Chi Minh City, Vietnam. A) Bilateral abnormal nonenhancing, confluent high signal in the periventricular and deep cerebral white matter of the high frontal parietal area and cerebellar hemispheres, thalamus, and pons. B) Almost complete resolution of abnormal findings 7 weeks later, after treatment.

week for seizure control. Repeated neurologic assessment on day 5 revealed normal eye movements and improved rigidity but total left-sided hemiplegia. The patient gradually improved over the next 4 weeks. A repeated MRI 7 weeks later (February 17) demonstrated almost complete resolution, with minimal residual white matter abnormalities (Figure, panel B). The patient was discharged for rehabilitation on February 21.

The diagnosis did not fit with dengue encephalitis because of a lack of CSF pleocytosis and the high protein levels; the presence of dengue IgM in the CSF was likely secondary to vascular disruption rather than intrathecal production. Review of the MRIs, which demonstrated early reversible white matter changes rather than delayed multifocal discrete lesions associated with ADEM, were diagnostic of dengue-associated posterior encephalopathy syndrome (PRES) (1).

PRES is an acute neurologic syndrome, typically in patients with blood pressure fluctuations or metabolic derangement (1). However, PRES has been recognized to complicate various infections accompanied by normal blood pressure $(2,3)$. Characteristic radiographic findings include bilateral white matter changes in areas supplied by the posterior circulation but can be diffuse, as described in this case, and resolve over weeks. High CSF protein levels correlate with cerebral edema and disease severity (4). The pathophysiology of PRES is thought to involve disruption to cerebral blood flow autoregulation, endothelial dysfunction, and vasogenic edema ( 1 ).

Most dengue infections cause a self-limiting febrile illness; however, life-threatening complications can occur, including increased capillary permeability, causing plasma leakage and shock. Like PRES, endothelial dysfunction is thought to underlie the capillary leak (5). Severe dengue can also occur with specific organ involvement (including neurologic) and without other severe features, as defined by the 2009 World Health Organization classification (6). Various neurologic manifestations have been described in dengue; however, PRES has been suspected in only 2 other reported cases $(7,8)$, possibly because of underreporting or misdiagnosis, especially given the limited access to neuroimaging services in dengue-endemic areas and the common assumption that PRES diagnosis requires hypertension or metabolic derangement to be present. Unlike ADEM, PRES usually only requires supportive treatment.

This case highlights the need to consider PRES in dengue patients with neurologic symptoms and that PRES should be distinguished from encephalitis or ADEM. The high CSF protein levels and characteristic MRI findings we have described could assist clinicians in dengue-endemic areas. 


\section{About the Author}

Dr. Mai is a neurologist specializing in infectious diseases of the central nervous system and serves as a clinical researcher at the Oxford University Clinical Research Unit in Vietnam and a clinician at the Hospital For Tropical Diseases in Ho Chi Minh City. Her research interests are clinical therapeutic trials of encephalitis, tuberculosis, and bacterial meningitis.

\section{References}

1. Fugate JE, Rabinstein AA. Posterior reversible encephalopathy syndrome: clinical and radiological manifestations, pathophysiology, and outstanding questions. Lancet Neurol. 2015;14:914-25. http://dx.doi.org/10.1016/S1474-4422 (15)00111-8

2. Bartynski WS, Boardman JF, Zeigler ZR, Shadduck RK, Lister J. Posterior reversible encephalopathy syndrome in infection, sepsis, and shock. AJNR Am J Neuroradiol. 2006;27:2179-90.

3. Ogunneye O, Hernandez-Montfort JA, Ogunneye Y, Ogu I, Landry D. Parainfluenza virus infection associated with posterior reversible encephalopathy syndrome: a case report.
J Med Case Reports. 2012;6:89. http://dx.doi.org/10.1186/17521947-6-89

4. Neeb L, Hoekstra J, Endres M, Siegerink B, Siebert E, Liman TG. Spectrum of cerebral spinal fluid findings in patients with posterior reversible encephalopathy syndrome. J Neurol. 2016;263:30-4. http://dx.doi.org/10.1007/s00415-015-7928-8

5. Yacoub S, Wertheim H, Simmons CP, Screaton G, Wills B. Microvascular and endothelial function for risk prediction in dengue: an observational study. Lancet. 2015;385(Suppl 1):S102. http://dx.doi.org/10.1016/S0140-6736(15)60417-2

6. World Health Organization. Dengue: guidelines for treatment, prevention and control. Geneva: World Health Organization; 2009.

7. Sohoni CA. Bilateral symmetrical parieto occipital involvement in dengue infection. Ann Indian Acad Neurol. 2015;18:358-9. http://dx.doi.org/10.4103/0972-2327.160096

8. Garg RK, Malhotra HS, Jain A, Kumar N. Dengue encephalopathy: very unusual neuroimaging findings. J Neurovirol. 2017;23:77982. http://dx.doi.org/10.1007/s13365-017-0547-7

Address for correspondence: Sophie Yacoub, Oxford University Clinical Research Unit, 764 Vo Van Kiet, Ho Chi Minh City, 0000, Vietnam; email: syacoub@imperial.ac.uk

\section{Relative Risk for Ehrlichiosis and Lyme Disease Where Vectors for Both Are Sympatric, Southeastern United States}

\section{Marcia E. Herman-Giddens}

Author affiliation: University of North Carolina, Chapel Hill, North Carolina, USA

DOI: https://doi.org/10.3201/eid2402.170962

To the Editor: The timely study on the relative risk for ehrlichiosis and Lyme disease in which the tick vectors, Amblyomma americanum and Ixodes scapularis, are sympatric notes that knowledge of tickborne diseases is "startlingly low" (1). The call for more research in diseases other than Lyme disease (LD) is long overdue. In the southeastern United States, 5 species of ticks bite humans (2). At least 11 associated human pathogens have been identified; all may cause tick paralysis $(2,3)$.

This study also prompts comment on drawbacks. First, even where $A$. americanum ticks outnumber $I$. scapularis in high-incidence LD areas (1), there is no mentioned concern about inflated LD case numbers resulting from reporting patients with erythema migrans (EM) from A. americanum tick bites (4). Second, there is no evidence for or against a 1:1 transmissibility factor.
Bites from infected ticks may not result in illness because of various factors. Subclinical cases may occur. Finally, LD may be reported more frequently because of EM occurrence compared with ehrlichiosis, which depends on laboratory criteria (5).

In addition, this study prompts pertinent observations. A. americanum ticks are known vectors of numerous pathogens and conditions, including several not yet reportable - for example, $\alpha$ gal allergy, Southern tick-associated rash illness, and Heartland virus - and no prevalence studies have been conducted, so their impact is unknown. It is notable that Monmouth County, New Jersey, USA, tests $I$. scapularis but not $A$. americanum ticks, which are more numerous, carry a greater number of pathogens, and are aggressive biters of humans.

Even though the Southeast United States has more tick species and tickborne pathogens, tick education campaigns, such as those conducted in the Northeast, are absent. The Southeast is experiencing human misery and economic impact from the increase in tick species and diseases. Attention to diseases other than LD is needed and is gratifying to see.

\section{References}

1. Egizi A, Fefferman NH, Jordan RA. Relative risk for ehrlichiosis and Lyme disease in an area where vectors for both are sympatric, New Jersey, USA. Emerg Infect Dis. 2017;23:939-45. http://dx.doi.org/10.3201/eid2306.160528

2. Stromdahl EY, Hickling GJ. Beyond Lyme: aetiology of tick-borne human diseases with emphasis on the southeastern United States. Zoonoses Public Health. 2012;59 